\title{
A Cirurgia Robótica nas Organizações Públicas de Saúde: 0 Caso do Instituto Nacional de Câncer (INCA)
}

\section{The Diffusion of Robotic Surgery in Health Public Organizations: The Case of the National Cancer Institute of Brazil (INCA)}

Claudio Pitassi

Doutorado em Administração, Professor Adjunto da Faculdades Ibmec, Brasil,

claudio.pitassi@gmail.com

http://lattes.cnpq.br/3151960182071356

Antonio Augusto Gonçalves

Doutorado em Engenharia de Produção, Professor Adjunto da Universidade Estácio de Sá, Brasil, augusto@inca.gov.br,

http://lattes.cnpq.br/1986689132934377

Jose Geraldo Pereira Barbosa

Doutorado em Administração, Professor Adjunto da Universidade Estácio de Sá, Brasil,

jose.geraldo@estacio.br,

http://lattes.cnpq.br/7648617323415171

Carlos Henrique Fernandes Martins

Mestrado profissional em Ciência Tecnologia Inovação em Saúde, Analista de C\&T Sr do Instituto Nacional do Câncer, Brasil,

CMartins@inca.gov.br,

http://lattes.cnpq.br/378242514613185

Resumo: O objetivo deste artigo é analisar o processo de implantação da cirurgia assistida por robótica no Sistema Único de Saúde (SUS) por meio de um estudo de caso realizado no Instituto Nacional de Câncer (INCA). Trata-se de um estudo teórico empírico com abordagem qualitativa, cujos dados foram coletados por meio de observação participante, documentos internos e entrevistas semiestruturadas. As evidências obtidas indicam a relevância do caso INCA para a incorporação definitiva da cirurgia robótica no SUS. Por um lado, relatos da área clínica não deixaram dúvidas dos benefícios da tecnologia para a qualidade de vida dos pacientes. Por outro, colheram-se indícios da falta de um consenso mais amplo sobre a relação custo-efetividade da robótica em hospitais públicos de países com o perfil social, econômico e demográfico brasileiro. Também há indícios de que o processo de adoção pelo SUS de tecnologias médicas de ponta não é uma decisão com critérios claramente definidos. Assim sendo, a inclusão dos procedimentos na tabela de remuneração revelou-se o grande desafio no processo de difusão da cirurgia robótica no SUS.

Palavras-chave: gestão em saúde; inovação; difusão; tecnologia; cirurgia robótica.

Abstract: The purpose of this article is to analyze the process of diffusion of surgery assisted by robotics in the Unified Health System (SUS) through a case study conducted at the National Cancer Institute of Brazil (INCA). This is a theoretical, empirical and qualitative study, whose data were collected through participant observation, internal documents and semi-structured interviews. The evidence collected indicates the relevance of INCA for the definitive incorporation of robotic surgery in SUS. Reports from the clinical area left no doubt of the benefits of robotic surgery to the quality of life of patients. However, indications were collected demonstrating the lack of consensus on the cost-effectiveness of robotic in public hospitals in countries with Brazilian social, economic and demographic profile. There are also indications that the process of adoption by SUS of state of the art medical technologies is not a decision with clearly defined criteria. Thus, the inclusion of procedures in the compensation table proved to be the major challenge in the diffusion process of robotic surgery into the SUS.

Keywords: health management; innovation; diffusion; technology; robotic surgery.

Texto completo em português: http://www.apgs.ufv.br Full text in Portuguese: http://www.apgs.ufv.br

\section{Introdução}

A aguda crise financeira atual e a queda na qualidade dos serviços de saúde pública evidenciam o esgotamento de uma visão fragmentada dos sistemas de saúde, centrada na remuneração da quantidade de serviços prestados por unidades isoladas, sem uma avaliação mais abrangente e integrada dos resultados das diferentes organizações públicas e privadas que compõem esse sistema (Porter \& Lee, 2013; Kaplan \& Porter, 2011).

As tentativas de atacar os problemas financeiros preferencialmente por meio do controle pontual dos custos dos procedimentos médicos geram um efeito negativo em espiral, com a redução ainda maior na qualidade dos serviços, a piora na saúde do paciente, que em casos como no tratamento do câncer, alcança estágios irreversíveis e, consequentemente, o aumento dos custos "escondidos" decorrentes das externalidades negativas em outras partes do sistema de saúde público (Kaplan \& Porter, 2011).

Há muito a inovação tecnológica é considerada a causa fundamental para o aumento da produtividade de países e para lucratividade das empresas (Schumpeter, 1934). No caso das organizações de saúde, particularmente os hospitais, a pressão oriunda de vários grupos cujos interesses muitas vezes são conflitantes (Hartley, 2005; Herzlinger, 2006; Windrum, Garcia-Goñi 
\& Fairhurst, 2010), a introdução pioneira de tecnologias médicas gera, em um primeiro momento, um agravamento dos problemas financeiros (Calil, 2011; Lara, 2015).

Ainda assim, é razoável supor que, no médio e longo prazo, esta adoção poderá contribuir para minimizar as taxas de erros médicos, tornando as organizações de saúde menos vulneráveis aos altos custos hospitalares decorrentes de infecções, lesões e até mesmo da morte de seus pacientes, o maior custo social de todos. Porém, no caso das organizações públicas, faz-se necessário encontrar mecanismos de financiamento dos investimentos em equipamentos de ponta, cujos custos não podem ser repassados imediatamente aos pacientes.

A cirurgia robótica, considerada neste artigo uma inovação em saúde em fase de difusão, é um novo campo de estudo na área de gestão de saúde, particularmente em sistemas públicos de países emergentes ou em desenvolvimento. Nas últimas décadas, uma grande variedade de robôs cirúrgicos foi implantada em hospitais ao redor do mundo, principalmente no EUA. A partir de meados da década de 2000, o uso da robótica em tratamentos médicos atingiu um crescimento vertiginoso nos hospitais de referência, apesar das incertezas ainda existentes (Barbash \& Glied, 2000).

No caso do Instituto Nacional de Câncer (INCA), os resultados obtidos até o momento da condução desta pesquisa indicaram que o uso do robô pode aumentar significativamente o custo do tratamento de câncer no Sistema Único de Saúde (SUS). A permanência deste quadro pode gerar resistências na introdução das mudanças organizacionais necessárias ao pleno usufruto dos benefícios da robótica, pois não há clareza sobre o custo-benefício da tecnologia. Sem critérios claros e objetivos para a precificação destes procedimentos na tabela do SUS, o uso da robótica não se consolidará como uma política pública ao alcance do cidadão comum.

Este estudo posiciona-se no campo da pesquisa de inovação em saúde pública e buscará responder à seguinte questão: como se dá a adoção da cirurgia robótica no contexto do SUS?Esta pesquisa amplia o conhecimento científico da área ao analisar o processo de difusão da cirurgia robótica no SUS, por meio de um estudo de caso realizado no INCA. A partir dos resultados obtidos, espera-se que pesquisadores e gestores tenham acesso a evidências que contribuam para o avanço na gestão da incorporação de tecnologias médicas de ponta na Saúde Pública Brasileira.

\section{Referencial teórico}

\subsection{Inovação em saúde}

Segundo o Manual de Oslo, da Organização para a Cooperação e Desenvolvimento Econômico (OCDE) inovação é a "implementação de um produto (bem ou serviço) novo ou significativamente melhorado, ou um novo processo, ou um novo método de marketing, ou um novo método organizacional nas práticas de negócios, na organização do local de trabalho ou nas relações externas" (OCDE, 2007, p.55). Para Freeman e Soete (1997), a inovação tecnológica altera as bases técnicas aplicadas à cadeia de valor, atuando sobre os direcionadores de custo e de diferenciação em busca de maior competitividade.
A inovação pode ser incremental ou disruptiva, dependendo do seu impacto transformador nas práticas usuais de um setor (Freeman \& Soete, 1997), sendo que, no caso da disruptiva, há um processo correlato de difusão da inovação até que esta se consolide, possibilitando então que se alcancem os benefícios sociais e econômicos esperados, o que geralmente ocorre após um longo período de tempo. Inovações incrementais tendem a ocorrer dentro de uma trajetória tecnológica dominante e as radicais podem criar novas rotas (Figueiredo, 2009).

De acordo com Rogers (2003), a difusão é o processo por meio do qual uma inovação é comunicada ao longo do tempo por meio de certos canais, aos membros de um sistema social. Trata-se de um tipo especial de comunicação, na qual as mensagens se referem a novas ideias, que possivelmente conflitam com as rotinas organizacionais estabelecidas. A difusão de novas ideias vem sofrendo profundas transformações em decorrência do caráter distribuído (geograficamente), fragmentado (em termos de disciplinas científicas requeridas em produtos e processos), e fluido (quanto à possibilidade de proteção) do conhecimento, exigindo um processo de especialização que força a passagem de processos e tecnologia para parceiros (Bell \& Figueiredo, 2012; Christensen, 2006).

Para que a difusão da nova tecnologia tenha maiores chances de sucesso é preciso que todos os departamentos da organização alinhem seus esforços ao longo do processo (Freeman e Soete, 1997). Assim sendo, inovar requer vender um conceito, fazendo com que a comunicação e o feedback tornem-se determinantes para o seu sucesso.

Segundo Fichman (2000), a intenção da organização de adotar uma tecnologia é influenciada por uma série de fatores, incluindo o custo da inovação, sua facilidade de utilização, a compatibilidade da tecnologia com os sistemas existentes e seu alinhamento com a estrutura organizacional.

Com relação às inovações em processos internos, Tidd et al. (2008) sugerem verificar: i) a possível obsolescência de equipamentos e sistemas em decorrência da inovação, especialmente se as instalações e o arranjo físico estão adequados; ii) os elementos infraestruturais, analisando se os sistemas de controle operacional e gerencial foram redesenhados; iii) as novas rotinas de trabalho, que poderão gerar a necessidade de novas competências em operação e manutenção.

Segundo Rogers (2003), há cinco características da inovação que afetam sua taxa de adoção: i) Vantagem relativa: grau em que uma inovação é percebida como melhor que o produto que ela substitui ou concorre; ii) Compatibilidade: grau em que uma inovação é percebida como sendo consistente com os valores, experiência e necessidades presentes dos adotantes potenciais; iii) Complexidade: grau em que uma inovação é percebida como sendo difícil de entender ou usar; iv) Possibilidade de ser experimentada: nível em que uma inovação pode ser experimentada, o que acarreta menores incertezas para os adotantes potenciais e permite o "aprender fazendo"; v) Visibilidade: grau em que os resultados de uma inovação são evidentes. 
A implantação de uma inovação na área de saúde deve ser iniciada no departamento ou área funcional onde a mesma foi demandada (Thakur, Hsu \& Fontenot, 2012). Segundo Levaggi, Moretto e Rebba (2009), o processo de tomada de decisão de investimentos em novas tecnologias hospitalares sofre grande influência dos médicos, os quais são reconhecidos como profissionais com elevadas competências técnicas, cujo foco prioritário está na saúde dos pacientes.

A partir da implantação, os resultados são avaliados e melhorias são realizadas, levando com que o novo processo ou tecnologia sejam gradualmente estendidos a outros. Herzlinger (2006) destaca seis forças relativas à inovação nos serviços hospitalares:

i) Financiamento da inovação que é uma força determinante de seu êxito ou fracasso;

ii) Regulamentação que ajuda a definir limitações e riscos de adoção e uso;

iii) Oportunidades de implantação de novas tecnologias, que se feitas tardiamente, poderão representar perda de competitividade;

iv) Poder decisório dos pacientes sobre a utilização de determinado material, medicamento ou nova tecnologia;

v) Responsabilização que requer que o inovador detenha a capacidade de comprovar a segurança e eficiência da inovação junto à sociedade e aos órgãos reguladores;

vi) Atores inseridos na área da Saúde são os principais protagonistas das inovações, tais como: seguradoras, hospitais, centros para tratamentos ambulatoriais, grupos defensores de pacientes e o corpo clínico e funcional.

Por este motivo, a colaboração entre os atores influencia significativamente a adoção e o uso de inovação em saúde (Powell et al., 2005), com destaque crescente para as práticas de inovação aberta (Hughes \& Wareham, 2010). Logo, a criação de significado nas relações interpessoais em redes de colaboração é frequentemente indicada como um requisito para uma organização inovadora em saúde (Ekedahl \& Wengstrom, 2008). Em linha com este argumento, Dixon-Woods et al. (2011) destacam que uma das formas mais eficazes para garantir a implantação de novas tecnologias médicas ou terapias é trabalhar em cooperação com os grupos de profissionais que devem participar da sua difusão.

Há evidências de que uma área bem dimensionada, capacitada e proativa de Engenharia Clínica (EC) influencia na adoção e utilização de uma nova tecnologia em serviços hospitalares (Freire et al., 2012). A introdução de tecnologias de ponta pode causar a alienação de conhecimentos antigos e requerer o desenvolvimento de novas habilidades, além do descarte de tecnologias consideradas incompatíveis com as mudanças, mas que ainda estejam em plenas condições de uso (Calil, 2011). Estes desafios são complexos devido às incertezas envolvidas, à necessidade de negociações políticas, às pressões de pacientes e profissionais de saúde, bem como à inércia organizacional e institucional (Daniels, 2006).

De acordo com Cecílio (2009), a gestão da saúde demanda uma perspectiva sistêmica, que envolve:

“...o conjunto de serviços de saúde, com suas diferentes funções e diferentes graus de incorporação tecnológica e os fluxos que se estabelecem entre eles. Tais fluxos serão definidos por protocolos, controlados por centrais de vagas ou de marcação de consulta, sempre na perspectiva de garantir o acesso dos usuários às tecnologias de cuidado de que necessitam, por meio da constituição e gestão de complexas "redes de cuidado" institucionais, operadas por intermédio de processos formais de referência e contrarreferência, que propiciem a circulação das pessoas por um conjunto articulado de serviços de saúde, de complexidades diferentes e complementares entre si." (Cecílio, 2009, p.549)

A crescente prevalência das doenças crônicas e nãocomunicáveis, tais como doenças cardiovasculares, câncer, doenças respiratórias e diabetes, resulta em um grande sofrimento humano, na perda de produtividade, na alta utilização dos serviços médicos e, nos modelos de gestão hoje predominantes, no descontrole dos custos de saúde (O'Donnell, 2014). Estes fatos explicam porque a gestão na área de saúde tem assumido um papel de grande relevância em todo o mundo, demandando modelos adequados a esta nova realidade. Em um cenário cada vez mais competitivo e de recursos escassos, as organizações de saúde, particularmente as públicas, necessitam urgentemente de novos instrumentos de avaliação (Cecílio, 2009)

Moraz et al. (2015) apontam que o financiamento da saúde é um assunto que suscita debates cada vez mais intensos, particularmente quanto à utilização e à alocação dos recursos. Nestes debates, fica claro que o emprego de recursos em uma nova tecnologia pode acarretar a restrição de recursos em outra área.

O novo paradigma da prática sanitária preconiza a adoção da Medicina Baseada em Evidência (MBE) (Maggio et al., 2016). Embora o processo decisório na MBE seja complexo e que inúmeros fatores técnicos, políticos, sociais, culturais e éticos estejam envolvidos, há uma crescente concordância a respeito da necessidade do emprego de evidências clínico epidemiológicas para auxiliar no processo de decisão no SUS. (Ministério da Saúde, 2008).

Segundo Silva (2003), a seleção de tecnologias médicas deve ter como objetivo tornar o sistema de saúde mais eficiente para população como um todo. Em 2010, a Política Nacional de Gestão de Tecnologias em Saúde definiu que a decisão de incorporar uma nova tecnologia no sistema deverá levar em consideração a evidência dos benefícios e dos custos correspondentes, quando comparada às tecnologias já existentes. Além disso, A gestão da saúde requer uma reflexão sobre os princípios da universalidade, integralidade e da equidade, visto que o SUS é um sistema hierarquizado, com um complexo processo decisório, que envolve diferentes necessidades e tetos financeiros. (Ministério da Saúde, 2010). Frisse (1999) aponta que as Tecnologias da Informação e Comunicação (TIC) podem ser um claro caminho para o avanço da gestão da saúde.

A implantação da cirurgia robótica pode ser considerada como um tipo de inovação disruptiva em serviços de saúde em fase de difusão, localizada na fronteira entre processos e inovação organizacional. O Quadro 1, adaptado de Varkey et al. (2008), apresenta um comparativo entre as recentes inovações em saúde: 
Quadro 1:Exemplos de inovação em saúde

\begin{tabular}{|l|l|l|}
\hline \multicolumn{1}{|c|}{ Tipo de Inovação } & \multicolumn{1}{|c|}{ Não Disruptiva } & \multicolumn{1}{c|}{ Disruptiva } \\
\hline Produto & Novos usos para drogas antigas & Ressonância Magnética, Tomografia Computadorizada \\
Processo & Telemedicina & PACS, Cirurgia Robótica \\
Organizacional & Clínicas Especializadas & Home Care \\
\hline
\end{tabular}

\subsection{Robótica na Saúde}

Devol, considerado o pai da robótica, patenteou em 1954 o primeiro manipulador com a memória de reprodução, evento que marcou o início da era da robótica moderna (Gibilisco, 1994). Desde então, os robôs têm sido utilizados em diferentes setores da indústria manufatureira contemporânea, particularmente na indústria automobilística, como atesta o desenvolvimento do sistema de produção denominado Toyotismo (Nof \& Hall, 2007). Na sua origem, associada à Revolução Digital, a robótica foi influenciada por duas grandes linhas de desenvolvimento científico: a cibernética, ou seja, homem e máquina trabalhando harmoniosamente juntos; e a inteligência artificial, robôs inteligentes que podem substituir totalmente a mente humana (Isaacson, 2014).

A utilização de robôs nas cirurgias em humanos é um fenômeno recente, que se intensifica a partir da segunda metade da década de 1990 (Giulianotti et al., 2003), embora, como destacam Ballantyne e Moll (2003), a cirurgia laparoscópica possa ser compreendida como uma "transição" para a cirurgia robótica. Como assinala Gomes (2011), a primeira cirurgia robótica foi realizada em 1985 no Memorial Medical Center, em Long Beach. Um robô industrial foi utilizado para colocar uma sonda durante a realização de uma biopsia no cérebro, usando a orientação de um tomógrafo computadorizado. Estudos recentes indicam que cerca de $50 \%$ das cirurgias passaram a usar robôs depois de apenas um ano da sua introdução nos hospitais (Nakib et al., 2013). O mesmo se observa no tratamento do câncer, o que reforça a necessidade de estudos a respeito da viabilidade econômica e da segurança dos pacientes (Baek et al., 2012).

Estudos têm comprovado os resultados positivos da cirurgia robótica para a qualidade de vida dos pacientes (Gaia et al., 2010), com destaque para: i) diminuição da perda de sangue; ii) o menor tempo de internação; iii) cicatrizes menores devido à não necessidade de incisões mutiladoras; iv) redução da dor e da necessidade de medicação prolongada; v) recuperação mais rápida e com menos complicações; vi) menor risco de infecção; vii) redução da necessidade de procedimentos adicionais. Já para os médicos, estudos demonstraram que a cirurgia robótica proporciona melhor visualização e permite movimentos mecânicos com maior grau de liberdade, além da diminuição da fadiga ou tensão nas articulações devido ao design ergonômico do robô (Barbash \& Glied, 2010).

Nas gerações mais recentes, o cirurgião realiza a cirurgia de um console remoto, que fornece imagens em 3D e alta definição, mais claras e com melhor detalhamento dos planos dos tecidos e da anatomia crítica. O envio eletrônico dos sinais dos joysticks do console para a ponta dos instrumentos imita os movimentos das mãos do cirurgião. Este recurso exclusivo permite que o cirurgião faça valer a sua "telepresença", podendo estar a quilômetros de distância do paciente. Estas características indicam algumas possibilidades que podem ser exploradas no desenvolvimento futuro da cirurgia robótica na área médica (Orvieto et al., 2011).

Mesmo considerando todos os benefícios, cumpre reconhecer que os referidos sistemas ainda têm custos fixos elevados quando comparados ao método tradicional, com preços variando de US $\$ 1$ milhão a US\$ 2,5 milhões por unidade. Também requerem manutenção dispendiosa e exigem a utilização de materiais de consumo adicionais, de uso específico em cada tipo de cirurgia. No entanto, estes custos podem ser compensados por meio de reduções nos custos de internação pós-operatória e por ganhos de produtividade, se os pacientes se recuperarem mais rapidamente (Barbash \& Glied, 2010). Em linha com a observação acima, Nakib et al. (2013) defendem que procedimentos robóticos podem ser considerados economicamente viáveis em uma configuração com alto volume de cirurgias e de tempos menores de internação hospitalar. O Quadro 2 apresenta as vantagens e desvantagens da utilização da cirurgia robótica, quando comparada à cirurgia tradicional:

\begin{tabular}{|c|c|c|}
\hline \multicolumn{3}{|c|}{ Quadro 2:Comparação entre cirurgia robótica e tradicional } \\
\hline & Cirurgia Robótica & Cirurgia Tradicional \\
\hline \multirow{4}{*}{ Vantagens } & Capacidade de Repetição & Versatilidade \\
\hline & Estabilidade e Acurácia & Experiência para julgamento \\
\hline & Destreza em escala milimétrica & Coordenação manual com a visão \\
\hline & Múltiplos sensores & Gerenciamento de várias atividades simultâneas \\
\hline \multirow{2}{*}{ Desvantagens } & $\begin{array}{l}\text { Incapacidade de processar informações } \\
\text { qualitativas }\end{array}$ & $\begin{array}{l}\text { Incapacidade de processar grandes volumes de } \\
\text { informações quantitativas }\end{array}$ \\
\hline & $\begin{array}{l}\text { Falta de Versatilidade } \\
\text { Alto custo }\end{array}$ & Imprecisão \\
\hline
\end{tabular}

Fonte: Adaptado de Camarillo, Krummel e Salisbury (2004).

Do ponto de vista das políticas públicas, cumpre reconhecer que o aumento das cirurgias robóticas nos hospitais públicos do país implicará na importação de um número elevado de equipamentos e periféricos, contribuindo para o crescimento do déficit existente na gestão do Complexo Industrial de Saúde, que ocorre, em grande parte, pela falta de capacidade de produção nacional de equipamentos médicos com maior conteúdo tecnológico (Button \& Oliveira, 2012). 


\section{Metodologia}

A pesquisa retratada neste artigo é um estudo teórico empírico com abordagem qualitativa, de natureza descritiva e exploratória (Miles, Hubermam \& Saldaña, 2014). Descritiva, porque relata as motivações para a implantação da cirurgia robótica no INCA. Exploratória, porque busca analisar a difusão da cirurgia robótica nos SUS, um processo ainda sem contornos definidos. O estudo adota o método de estudo de caso único (Yin, 2005), tendo como principais formas de coleta de dados a observação participante, documentos internos e sete entrevistas semiestruturadas.

O estudo de caso, na medida em que considera as dimensões de tempo e espaço (Yin, 2005), mostrou-se uma ferramenta metodológica adequada à análise de um fenômeno cuja evolução se dava concomitantemente às profundas mudanças estruturais que ocorriam no INCA a partir da segunda metade da década de 1990, que forçavam a Instituição a conciliar os objetivos assistenciais, com a busca pela vanguarda na pesquisa científica em câncer na América Latina. Os efeitos deste contexto ganham ainda mais relevância em função dos contingenciamentos orçamentários por que passava o INCA nos anos de 2014 e 2015, fato que impunha sérias restrições ao crescimento da cirurgia robótica na Instituição.

A escolha de casos emblemáticos é um procedimento científico adequado em função da oportunidade de se entender dinâmicas específicas, mas que podem trazer importantes lições para outras organizações (Miles, Hubermam \& Saldaña, 2014). A escolha do INCA como caso único justifica-se pelo esforço da organização em introduzir inovações tecnológicas de ponta e pelo fato de o INCA ser, na época da realização desta pesquisa, a primeira instituição pública de saúde a usar a cirurgia robótica, cujos desdobramentos influenciariam, nas palavras dos próprios gestores, na decisão de incluir os procedimentos cirúrgicos realizados por meio da robótica na tabela de remuneração do SUS.
Os dados foram coletados por meio de observação participante, documentos internos e entrevistas semiestruturadas. A observação participante é considerada uma fonte importante para a condução de pesquisas científicas (Argyris, Putnam \& Smith, 1985), particularmente em organizações de saúde (Queiroz et al., 2007). No caso, dois autores deste artigo, gestores do INCA, por fazerem parte do grupo social que vivenciou a introdução da cirurgia robótica ao longo de quase três anos, tiveram uma observação participante que propiciou: i) identificar os sujeitos chave e obter espaço nas agendas dos profissionais de saúde, em geral, muito ocupados; ii) Dispor de um conhecimento mais refinado da linguagem, cultura $e$ regras da Instituição e do seu contexto, particularmente quanto à obediência ou transgressão destas regras e das tensões no relacionamento com o Ministério da Saúde (MS); iii) identificar as motivações e os sentimentos verbalizados pelos participantes, possibilitando separar nas falas os aspectos emocionais, fortemente afetados pela conjuntura de crise e de alternância de poder por que passava o INCA, dos aspectos fundamentais para os objetivos da pesquisa.

Ao longo do segundo semestre de 2014 , os pesquisadores realizaram seis entrevistas com os responsáveis pela introdução $e$ uso pioneiro da cirurgia robótica na organização. As entrevistas foram apoiadas em um roteiro com perguntas abertas por meio do qual se buscou coletar evidências do impacto da cirurgia robótica no INCA. Na etapa exploratória, as respostas obtidas foram tratadas pelo método de análise de conteúdo do tipo temático ou categorial (Bardin, 1999). As categorias de análise foram definidas a partir da revisão da literatura. Durante o ano de 2015 a análise dos dados revelou a necessidade de se aprofundar a discussão sobre a precificação dos procedimentos da cirurgia robótica, o que foi tratado em uma nova entrevista. O Quadro 3 apresenta a relação dos entrevistados:

Quadro 3: Sujeitos da Pesquisa

\begin{tabular}{|c|c|c|c|}
\hline Entrevistado & Área funcional & Código & Responsabilidades em relação ao sistema robótico \\
\hline $\begin{array}{l}\text { Coordenador de Assistência } \\
\text { (COAS) }\end{array}$ & COAS & E1 & $\begin{array}{l}\text { Coordenador da COAS, cujos médicos lideraram a solicitação de aquisição do sistema } \\
\text { robótico. }\end{array}$ \\
\hline $\begin{array}{l}\text { Chefe Clínica Cabeça e } \\
\text { Pescoço }\end{array}$ & HC1 COAS & E2 & Atuou como o "campeão do projeto", liderando as solicitações para a compra do robô. \\
\hline Chefe Clínica Abdômen & HC1 COAS & E3 & Um dos três médicos que participaram da equipe que recebeu treinamento nos USA. \\
\hline Enfermeira & HC1 COAS & E4 & $\begin{array}{l}\text { Selecionada para a equipe que foi para o EUA e apoiou a todas as cirurgias robóticas } \\
\text { realizadas no INCA. }\end{array}$ \\
\hline Gestor da EC & $\begin{array}{l}\text { Coordenação de Adm. } \\
\text { Geral }\end{array}$ & E5 & Manutenção preventiva e primeiro combate nas falhas recuperáveis. \\
\hline Gestor da TIC & DTI & E6 & Desenvolvimento de sistemas de informação. \\
\hline Assessora & COAS & E7 & $\begin{array}{l}\text { Responsável pelos estudos de custo/benefício e cus } \\
\text { implantação. }\end{array}$ \\
\hline
\end{tabular}

Fonte: Elaboração própria.

A Figura 1 apresenta uma estrutura simplificada do INCA, demarcando em negrito as áreas em foco neste artigo. As unidades centrais de análise são as responsáveis pela adoção das cirurgias robóticas no tratamento oncológico, conduzidas pelo corpo clínico da Coordenação de Assistência e apoiados pela Engenharia Biomédica. 
Figura 1: Organograma simplificado do INCA

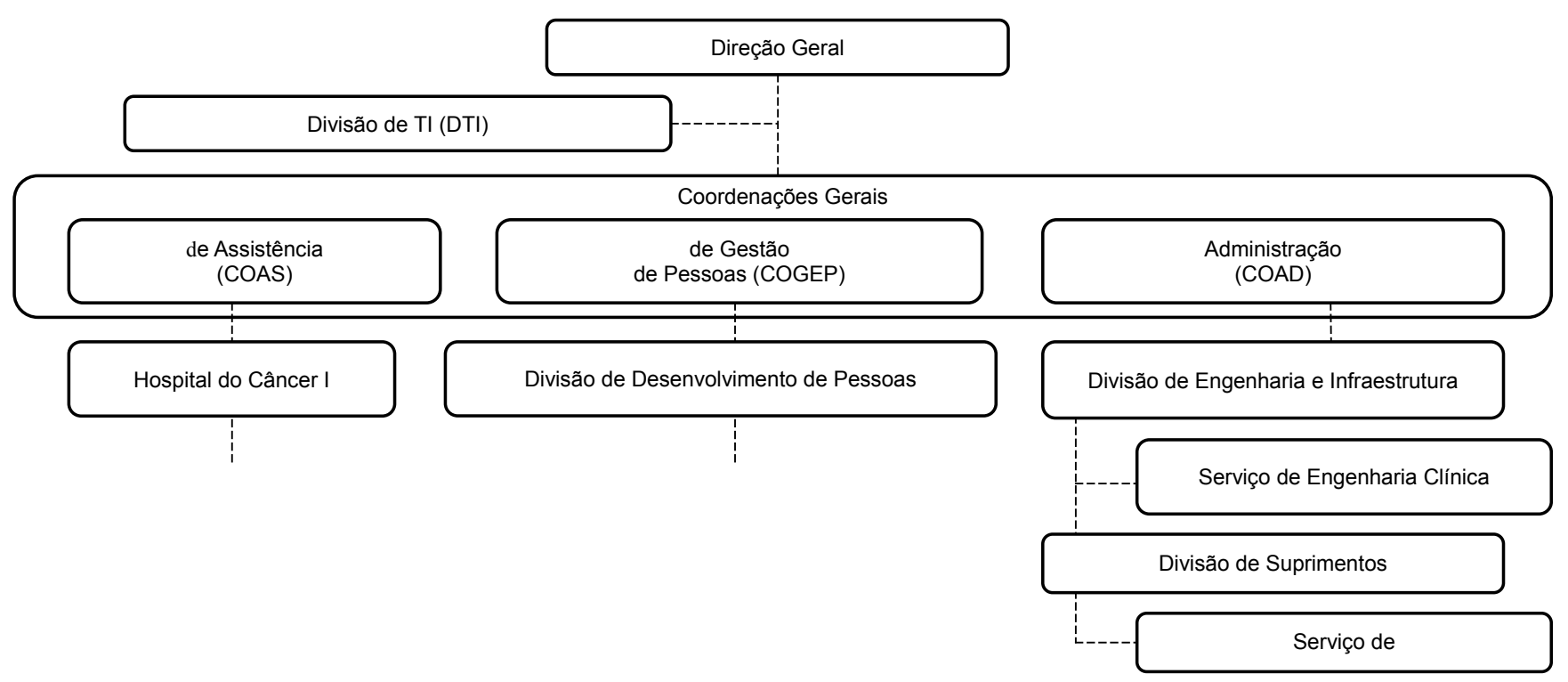

Fonte: Elaboração própria.

\section{O caso INCA - Implantação do Sistema de Cirurgia Robótica DA VINCI}

No Brasil, o INCA é um órgão do Ministério da Saúde que tem por responsabilidades a coordenação e o desenvolvimento de ações para a prevenção e controle na classe terapêutica de oncologia. Suas unidades hospitalares, localizadas no município do Rio de Janeiro, integram o SUS e oferecem tratamento integral às pessoas com câncer. $O$ Instituto também desenvolve atividades de pesquisa científica de ponta, particularmente nas pesquisas clínicas das grandes farmacêuticas globais, nas quais se destaca como a mais importante instituição da América Latina.

Em janeiro de 2012, o INCA adquiriu da empresa americana Intuitive Surgical (http://www.intuitivesurgical.com) o robô cirúrgico da Vinci@ SI, atualmente instalado no centro cirúrgico do Hospital do Câncer I. A solicitação de compra partiu da equipe médica de Cabeça e Pescoço e, para decidir pela incorporação ou não da tecnologia, foi realizado um estudo de viabilidade, que durou dois anos e meio e contou com a participação da Coordenação de Programas de Pós-graduação em Engenharia (Coppe) da Universidade Federal do Rio de Janeiros (UFRJ). Uma vez adquirido o robô, a Intuitive ficou responsável pelos treinamentos da equipe de implantação.

A Intuitive detinha a patente do robô cirúrgico, que expiraria em 2015. Na época da realização desta pesquisa o INCA tinha informações vagas sobre a existência de outros dois fabricantes esperando a queda da patente para entrar no mercado, no entanto, os entrevistados não demostravam muita preocupação com o assunto, como atesta a fala a seguir: "Nos congressos mundiais que nós frequentamos existe a informação em off de que outras máquinas estão sendo desenvolvidas, coreanas e outras americanas, canadenses, mas nada muito assertivo" (E3).

Segundo o representante da Engenharia Clínica (EC), a Intuitive adotava uma estratégia agressiva de aquisições, inclusive de empresa concorrentes, o que poderia influenciar negativamente nas futuras quedas de preço. Já para os médicos, havia a expectativa de que uma maior concorrência no futuro acarretasse a queda nos preços: "Mas com certeza outras máquinas virão...naturalmente a competição entre elas trará uma redução no custo de aquisição desses devices, desses elementos que a gente usa na cirurgia robótica" (E3).

O Sistema Cirúrgico da Vinci® é um sistema robótico do tipo mestre-escravo, no qual o cirurgião opera em um console remoto (mestre), controlando diretamente o movimento dos braços robóticos (escravo), que contêm os instrumentos usados na cirurgia, na sala de operações. O carro do paciente é a mesa de operação com robô, composto por quatro braços poliarticulados, sendo três braços para os instrumentos e um braço para a câmera endoscópica, com flexibilidade de $360^{\circ}$ graus e movimentos precisos. Ao cirurgião é fornecido um console, com um visor estereoscópico (visão em 3D de alta definição), que é colocado juntamente com os manipuladores "mestres" anatômicos de controle de alças (interfaces táteis), que controlam diretamente os movimentos dos braços robóticos "escravos", dentro do corpo do paciente.

A solução adquirida vinha com dois consoles, o que permitia a cirurgia em que dois cirurgiões operavam em conjunto, o que era prática comum, corroborando com o critério de colaboração destacado por Ekedhal (2008), para a identificação de uma organização inovadora em saúde. A fala seguinte destaca a prática: "A maioria das vezes eu estou com os três médicos presentes na clínica...às vezes um entra para ajudar...não tem isso de só um que vai ficar não. O outro entra, ajudam entre eles, cooperam" (E4).

O cirurgião pode controlar a posição do endoscópio, dar zoom e girar o campo de visão a partir do console. Os instrumentos têm sete graus de movimento que imitam a destreza da mão humana. Cada instrumento tem uma tarefa cirúrgica específica, como fixação, sutura e manipulação de tecidos. Dadas às características observadas, pode-se afirmar que, no estágio atual, os sistemas 
robóticos, com crescente utilização na medicina, associam-se ao conceito de cibernética e não de inteligência artificial, conforme a diferença discutida em Isaacson (2014).

O INCA foi a primeira Instituição Pública brasileira na área médica a ter o robô cirúrgico. Hoje, o INCA já é uma referência na área, sendo inclusive responsável pelo treinamento de médicos oriundos de outros hospitais públicos, tais como o Hospital Marcílio Dias. Nos casos em que o INCA já dominava os procedimentos cirúrgicos, a própria equipe do INCA treinava os demais profissionais. Essa prática ajudava a organização a contornar as restrições orçamentárias vigentes à época e estimulava a cooperação entre os profissionais da Instituição, em linha com o argumento de Dixon-Woods et al. (2011).

Uma das exigências aos profissionais que utilizariam o robô era a fluência em inglês, já que a equipe teria que fazer um treinamento, cujo custo chegava a 10 mil dólares por pessoa, nos EUA, sede do fabricante. Foram treinados oito cirurgiões, seis inicialmente, dois de cada especialidade (Cabeça e Pescoço, Abdômen e Urologia) e posteriormente dois em Ginecologia. Os treinamentos tinham uma fase inicial na WEB, depois em um módulo simulador e, por fim, os profissionais eram enviados para um treinamento presencial de três meses nos EUA. No momento da realização da pesquisa, a restrição orçamentária, já mencionada, limitou severamente as verbas para este treinamento, impedindo um acesso mais amplo do corpo médico ao uso do robô.

Para que o cirurgião encontre-se apto para se tornar instrutor, são necessárias ao menos a realização de 50 cirurgias, conforme cita um dos entrevistados:

“... nossa curva de aprendizado requer ao menos 50 cirurgias realizadas. Neste instante nós recebemos um certificado do programa do fabricante do robô, ganha um grau de mestre, de ser instrutor de outros cirurgiões" (E3).

As motivações para a introdução pioneira da cirurgia robótica no INCA evidenciam o papel de vanguarda da Instituição na pesquisa e tratamento do câncer, no Sistema Público de Saúde, o que indica, de acordo com o que afirma Rogers (2003), que a visibilidade da Instituição pode ter afetado a decisão de adoção, como evidencia a narrativa a seguir:

"Oferecer o atendimento para doentes oncológicos no mais alto padrão técnico, médico, humano possível e imaginável... Se você proporciona uma tecnologia médica que não é oferecida em nenhum outro lugar, você está colocando a sua instituição lá em cima. Esse foi sempre um orgulho dos que trabalham aqui... A possibilidade de oferecer para doentes do SUS uma tecnologia que ainda não era disponível para doentes a nível privado" (E2).

Nas entrevistas ficou claro que os critérios de avaliação percebidos pelos gestores da Instituição são diferentes daqueles usualmente adotados para decidir sobre a incorporação de tecnologias em hospitais privados, como evidencia o depoimento abaixo, no qual se destaca o papel do INCA na definição de critérios para o SUS:

"[...] é uma obrigação do Instituto estar na frente, testando novos procedimentos..e preparando para poder dar resposta ao SUS se essas novas tecnologias são importantes para o sistema... Então, mais daqui há 2 anos a gente vai poder ver esse procedimento incorporado na tabela do SUS (E1)."
Para os médicos especialistas, todos com elevada reputação em suas áreas, a introdução da cirurgia robótica no INCA era vista como uma oportunidade única de aprendizagem de novas tecnologias. A fala abaixo, confirmando o argumento de Levaggi, Moretto e Rebba (2007), traz indícios do papel protagonista dos médicos nas incorporações de tecnologias em hospitais públicos com as características do INCA:

"[...] na minha opinião, foi um dos grandes ganhos do Projeto Robótica. Você sabe que um dos atrativos do profissional trabalhar em uma instituição, principalmente em uma instituição pública, onde a remuneração não é atrativa, é a possibilidade deste cirurgião executar os procedimentos no mais alto nível e, ainda, ser capaz de desenvolver e aprender novas tecnologias" (E2).

Em linha com o que destacaram Gaia et al. (2010), os ganhos para os pacientes vis-à-vis aos procedimentos convencionais em termos de conforto e qualidade de vida, tais como diminuição do tempo de internação, cortes menores, menos sangramento, reabilitação mais rápida, redução do risco de infecção e menos complicações, foram ressaltados por todos os médicos entrevistados, o que, segundo o argumento de vantagem relativa de Rogers (2003), estimulou a implantação da nova tecnologia na organização. Os depoimentos também corroboraram o argumento de Levaggi, Moretto e Rebba (2007) de que o foco na melhora na saúde do paciente é priorizado nas avaliações dos médicos sobre os benefícios de incorporações tecnológicas.

“... a cirurgia robótica diminui dramaticamente o tempo de internação, diminui dramaticamente as complicações e reabilita o doente de uma forma muito mais rápida" (E2).

"Nossos resultados são semelhantes ou iguais aos obtidos pelos grupos norte americanos ou europeus... Os resultados são rigorosamente semelhantes, as vantagens que são apregoadas para os doentes de lá foram obtidas nos pacientes daqui" (E2)

"Para cirurgia do câncer do reto, a cirurgia robótica veio trazer um avanço muito grande na preservação esfincteriana desses doentes, que na cirurgia convencional se curavam do câncer, mas herdavam complicações dessas cirurgias, com a lesão de nervos. Hoje, com a cirurgia robótica, com a precisão, pela qualidade da imagem, nós conseguimos fazer essa preservação nervosa e, consequentemente, a preservação das funções esfincterianas" (E3).

Apesar dos benefícios para os pacientes, as evidências coletadas indicam divergências sobre a decisão de adotar definitivamente a cirurgia robótica no INCA. Os depoimentos abaixo deixam clara a diferença de entendimento entre as áreas clínicas (benefícios) e as áreas técnicas (custos), o que, segundo o argumento de Freeman e Soete (1997) em defesa do alinhamento de expectativas e convergência de significados, pode impactar negativamente a implantação da cirurgia robótica na Instituição:

"A questão de ele ter alta mais cedo, eu acho que é a questão mais importante nessa história toda... Você imagina, se tem um Sistema (de Saúde) em que um dos gargalos é o leito do paciente, quer dizer, o doente não está podendo ser tratado porque não tem leito vazio para ele operar. Se eu instituo uma nova modalidade cirúrgica em que o doente, ao invés de ficar 15 dias, ele vai ficar 48 horas, quanto vale o ganho desses leitos que eu estou fornecendo a mais?" (E2).

"Então, a área privada ela tem um interesse diferente da área pública. Nós entendemos que na área pública estudos ainda devem ser desenvolvidos para se decidir sobre a incorporação ou não. Estudos clínicos, no sentido de se 
identificar o seguinte: qual o custo-benefício desta tecnologia? Qual o custo efetividade? Ela traz resultados para o paciente? Ou seja, dentro da área pública eu entendo que os pacientes estão começando a ser tratados agora, então você ainda não tem um horizonte de tempo que possibilite olhar os resultados" (E5).

Contribuía para a falta de clareza a respeito do custo-benefício da cirurgia robótica o fato de o perfil sócio, demográfico e cultural do paciente da rede pública ser diferente do mesmo perfil do paciente da rede privada. No último caso, o paciente provavelmente tem maior acesso à medicina preventiva, o que permitiria detectar a doença em seus estágios iniciais, quando a cirurgia robótica é mais efetiva. Em estágios avançados, talvez não haja outro recurso senão utilizar a cirurgia aberta. Tomando por base o critério de visibilidade proposto por Rogers (2003), essa diferença pode fazer com que a cirurgia robótica mostre-se mais atrativa em hospitais privados do que em hospitais públicos que atendem ao SUS, como é o caso do INCA.

Como fatores que contribuíram para que o INCA incorporasse a cirurgia robótica, os gestores entrevistados destacaram a qualidade da equipe de profissionais e a existência de estruturas dedicadas ao robô, particularmente da EC, corroborando o que foi destacado na literatura a respeito da incorporação de tecnologias em hospitais (Freire et al., 2011). O depoimento abaixo destaca esses pontos:

"O INCA ele realmente tem uma equipe de pessoas que é um diferencial. Se a gente for começar pelo corpo clínico, realmente tem pessoas que são referência na representação dos seus setores. O INCA também montou uma estrutura de engenharia, claro não só para cirurgia robótica, mas para todo o parque do INCA... Nós visitamos várias instituições, instituições privadas. Estivemos recentemente em São Paulo visitando algumas instituições que não possuem uma estrutura de engenharia que o INCA possui" (E5).

No momento da realização da pesquisa, a EC não dispunha de uma estrutura voltada exclusivamente para suporte ao sistema robótico. A área de assistência técnica atuava como fiscal do contrato. Quando indagado sobre as condições que garantiriam o alcance da meta arrojada de nível de serviço acordada com a área médica, sem contar com uma área totalmente dedicada ao robô, o mesmo gestor explicou as características específicas da relação com o fornecedor da tecnologia robótica:

"Essa máquina é monitorada remotamente. Ela está conectada numa rede de computador, onde é monitorada diretamente pelo fabricante nos Estados Unidos. Qualquer desvio que haja no comportamento desse equipamento, segundo o fabricante, ele consegue identificar e se antecipar a possíveis paradas. Por isso é que ele pactuou essa meta com a gente" (E5).

As evidências coletadas indicam que a relação da EC com o fabricante do robô era diferente da relação adotada com os fabricantes de outros equipamentos. Isso pode indicar que, em conformidade com o argumento de Bell e Figueiredo (2012) e Chistenssen (2006) sobre a divisão do trabalho social decorrente da penetração da ciência em produtos e processos, o que ocorreu com a robótica na área industrial também pode estar ocorrendo na área médica. O depoimento a seguir traz evidências da capacidade de absorver os conhecimentos embutidos nos softwares do robô:

"O corpo técnico do INCA nunca vai conseguir dominar todas as tecnologias médicas, é um universo muito grande. Atualmente existe no INCA mais de 7.000 equipamentos instalados. Então, a Engenharia Clínica não tem a capacidade de conhecer tudo profundamente. $O$ que a Engenharia Clínica do INCA hoje faz é se ocupar de ações estratégicas" (E5).

Quando indagado sobre as diferenças entre a estratégia de absorção da tecnologia robótica, quando comparada às outras tecnologias de alta complexidade, o gestor da EC não deixou claro os critérios que explicam essa diferença, o que pode indicar a necessidade de maior aproximação das áreas técnicas nas decisões de incorporação de tecnologias nos sistemas públicos de saúde:

"Eu não sei que critérios foram adotados na época para o sim ou para o não. Atualmente não é previsto... Sim, nós temos equipamentos que a equipe de Engenharia Clínica entendeu que era interessante... Para o sistema robótico, não" (E5).

O centro cirúrgico contava com sete enfermeiras atuando nos turnos da manhã e da noite. O objetivo era que todas elas estivessem capacitadas para apoiar os médicos no uso do robô. As operações eram realizadas por especialidades em cada dia da semana: segunda-feira, gineco; terça-feira, cabeça e pescoço; quarta-feira, urologia; na quinta-feira, abdômen, etc. A próxima especialidade programada para usar a robótica era a de tórax. Esta programação fazia com que o médico realizasse no máximo uma cirurgia por dia e impunha restrições para um aumento de efetivo, já que isso acarretaria uma ociosidade muito grande dos médicos nos outros dias da semana, ao menos no que tange às cirurgias robóticas.

A tendência de restrição de recursos apontada por Moraz et al. (2015) pode explicar porque praticamente todos os entrevistados disseram que a grande barreira era o alto custo, o que é ainda mais relevante por se tratar de uma instituição pública subordinada ao Ministério da Saúde, que precisa tomar a melhor decisão sobre a alocação de recursos, como deixa claro o depoimento seguinte: "Primeiro o custo. Não é barato, a tecnologia é cara. Então, nos moldes de um hospital como o Inca, fica difícil você justificar a compra de um aparelho desses" (E3).

Apesar de o robô estar teoricamente disponível 24 horas por dia, dentro da meta de $98 \%$ de disponibilidade, seu uso era definido pelas demandas da área médica. $\mathrm{Na}$ época da realização desta pesquisa, o INCA realizava apenas uma cirurgia por dia. Uma das barreiras para aumentar o número de cirurgias por dia era o número limitado de pinças, que impedia que outra cirurgia fosse realizada enquanto as pinças usadas anteriormente não fossem limpas. Segundo o gestor da EC, um dos motivos para que o INCA não investisse agora em um arsenal maior de periféricos, tais como óticas e pinças, seria o fato, já apontado anteriormente, de falta de informações sobre a relação custo/benefício.

A importação de equipamentos periféricos foi apontada como um problema, ainda mais grave em função da forte valorização 
cambial observada no país a partir de 2014. Também foram relatados problemas frequentes com a Agência Nacional de Vigilância Sanitária (ANVISA) para a liberação dos equipamentos importados. É importante ter em mente que a importação desses equipamentos contribui negativamente para o déficit crônico que o Brasil vem apresentando, ao longo das últimas décadas, no Complexo Industrial da Saúde (CIS), conforme apontado por Button e Oliveira (2012).

No que se refere aos hospitais particulares, inclusive em relação aos planos de saúde, o paciente decidia se queria usar a cirurgia robótica e pagava pela diferença em relação às coberturas acordadas, como explica o depoimento a seguir. "Ele quer cirurgia robótica, então o custo vai para o paciente. Então, no particular tem um jeito mais simples que é você repassar uma parte do custo" (E7). Como ainda não havia um valor e um código para a cobrança deste procedimento no SUS, não era possível atender às demandas do SUS nos hospitais privados.

A criação da Comissão Nacional de Incorporação de Tecnologias no SUS (CONITEC) em 2011, bem como do Departamento de Gestão e Incorporação de Tecnologias em Saúde (DGITS), em agosto de 2013, ambos ligados ao MS, indicavam que a incorporação da robótica deveria ser discutida nessas instâncias decisórias. Os depoimentos coletados no INCA não permitiram entender em que momento a análise da incorporação da cirurgia robótica deveria passar das instâncias técnicas supracitados para o CONITEC, mas a pesquisa realizada no site deste órgão no mês de setembro de 2015 não indicava que a cirurgia robótica estivesse em análise.

É importante frisar que o fato do INCA não usar os custos apurados na cirurgia para elaborar o seu orçamento, o qual era definido em função das prioridades estabelecidas pelo MS, pode dificultar o pleno entendimento da avaliação econômica de novas tecnologias. Evidências colhidas indicaram que o INCA enxergava a necessidade de uma maior objetividade na apuração dos custos dos procedimentos de alta complexidade: "Nós temos que minimamente tentar equiparar isso... até para termos uma efetiva equiparação do que nós produzimos com o que é vinculado aos nossos sistemas, independente de isso ser um orçamento ou não" (E7).

Como anteriormente mencionado, a falta de parâmetros para o pagamento impede a disseminação dos procedimentos apoiados em cirurgia robótica no SUS. Entretanto, se o MS continuar adotando os procedimentos de precificação tendo por base os critérios criticados por Porter e Lee (2013), ou seja, sem considerar as externalidades positivas obtidas em todo o sistema de saúde, é possível que a introdução da cirurgia robótica, de maior complexidade tecnológica, tenda a agravar os problemas de remuneração na cadeia de suprimentos da saúde, piorando ainda mais a situação financeira do sistema.

Com vistas a capturar o potencial das TIC conforme apontado por Frisse (1999), a DTI desenvolveu um sistema de informação (SI) para registrar todas as cirurgias robóticas. As informações colhidas pelo SI permitiu que os casos cirúrgicos fossem avaliados retrospectivamente, considerando o sexo, a idade, o peso, as indicações para cirurgia, os tempos de montagem e operatório, as complicações, a taxa de conversão para o procedimento laparoscópico, os dias de internação. Em linha com os resultados obtidos com o desenvolvimento do Banco Nacional de Tumores (Gonçalves, Pitassi e Moreno, 2014), a DTI preparava-se para aumentar sua contribuição para a pesquisa científica, abrindo oportunidades para o maior uso das informações geradas pelas cirurgias robóticas nas pesquisas clínicas, como pode ser visto na narrativa a seguir:

"Este cenário será alterado com a chegada dos novos servidores aprovados no concurso de 2015. Com estes colaboradores, será criada a área de Gestão da Inovação e Governança em TI, justamente para promover uma maior integração das áreas de TIC com a Pesquisa e Assistência em projetos de inovação dessa magnitude (a robótica) (E6)."

\section{Considerações conclusivas}

Em resposta à questão de pesquisa formulada, os autores deste artigo entendem que a análise do caso permitiu trazer à tona uma rica descrição do processo de difusão da cirurgia robótica no INCA. O caso trouxe evidência de que os procedimentos cirúrgicos assistidos por robô proporcionam resultados substancialmente melhores para a qualidade de vida dos pacientes com câncer. Pode-se mesmo afirmar que a cirurgia robótica está revolucionando a maneira de operar nesta área, principalmente nos procedimentos mais complexos como cabeça e pescoço, trazendo inovações radicais nos processos, que permitem superar os limites da cirurgia tradicional.

A triangulação dos documentos, entrevistas e observação participante, trouxe à tona pontos que precisam ser melhor explorados em pesquisas futuras. No que diz respeito à incorporação da cirurgia robótica no INCA, os relatos obtidos trazem evidências de que a compra da tecnologia pode ter sido influenciada principalmente por profissionais reconhecidos no INCA, que buscavam manter sua alta qualificação técnica e a reputação da Instituição, e não como decorrência de uma necessidade do SUS perante a evolução tecnológica do sistema de saúde privado. Também há indícios de que a cirurgia robótica pode ter contribuído para reter talentos médicos, desmotivados pelas reduções de investimento impostas pela crise orçamentária por que passava o Estado Brasileiro nos anos em que esta pesquisa foi realizada. De fato, há evidências de que a introdução do robô teve um grande efeito motivacional sobre os médicos envolvidos.

Documentos internos comprovam que a decisão de incorporação foi apoiada em um estudo técnico elaborado pela COPPE. No estágio atual, o caso trouxe evidências de que ainda não há vinculação direta entre os custos gerados por este tipo de cirurgia e o orçamento da Instituição, o que dificulta a adoção de técnicas e métodos de custeio adequados. A relação do INCA com os órgãos responsáveis pela definição de custos de procedimentos na tabela do SUS não foi detalhada pelos entrevistados. Os dados coletados indicaram que a decisão de incorporação tecnológica está concentrada na cúpula do MS e pode decorrer de pressões políticas e do lobby dos fabricantes, em vez de critérios puramente técnicos. A percepção dos funcionários do INCA, que consideram o 
Instituto um centro de excelência com forte influência nas decisões relativas à incorporação tecnológica na classe terapêutica de câncer, ainda não corresponde ao orçamento que é destinado pelo MS para este fim.

O caso também trouxe evidências de que a introdução dos sistemas robóticos cirúrgicos acarreta incertezas que podem retardar a incorporação generalizada dessa nova tecnologia na saúde pública brasileira. Uma grande barreira são os custos fixos de aquisição do robô e, principalmente, os custos variáveis de compras dos periféricos e insumos. Também são expressivos os custos de treinamento dos médicos, já que ainda é necessário enviar os cirurgiões para treinamento nos EUA. Cabe ressaltar que este custo poderá ser reduzido se o treinamento for ministrado pelos próprios cirurgiões do Instituto, que precisam em média cerca de 50 cirurgias assistidas por robô para o pleno domínio da tecnologia.

No caso dos hospitais particulares, o uso de robôs é um processo irreversível. Nestes hospitais a regra é clara: quem pode, paga. Já no contexto do SUS, dadas as diferenças de perfil socioeconômico do paciente, parece prematuro concluir que a abordagem robótica em câncer forneça resultados amplamente superiores em comparação com a cirurgia laparoscópica e aberta tradicional, o que justifica a necessidade, apontados pela EC, de se aprofundar os estudos sobre o uso desta tecnologia na saúde pública. Mais do que isso, dada a precariedade atual do sistema publico de saúde brasileiro, e dada a existência de necessidades básicas de tratamento dos pacientes, os relatos obtidos deixaram dúvidas se os investimentos em alta tecnologia são econômica e socialmente justificáveis para o SUS.

Estudos prospectivos comparativos poderão ajudar a delinear melhor as vantagens deste tipo de incorporação tecnológica para a saúde pública brasileira. No caso estudado, houve indício de que a falta de consenso sobre a relação custo/benefício da cirurgia robótica tem atrasado decisões relativamente simples, que poderiam contribuir para a rápida absorção dos custos fixos, tal como a compra de kits de periféricos sobressalentes. A situação foi agravada pela conjuntura de restrição orçamentária por que passava o órgão nos anos de 2014 e 2015.

O estudo de caso indicou que os principais impactos organizacionais da implantação da robótica nos hospitais públicos podem ser assim resumidos:

processos - identificação sistemática dos casos que justificam os procedimentos assistidos por robô, conciliando todos os interesses envolvidos, principalmente os dos doentes preteridos e seu familiares;

ii) estrutura - no modelo de negócios adotado pelo INCA as manutenções mais complexas ficavam a cargo da Intuitive. Ainda assim, o uso de tecnologias de alta complexidade, como é o caso dos sistemas robóticos, exige uma área de EC adequadamente dimensionada e capacitada;

iii) pessoas - o acesso às novas tecnologias revelou-se uma das grandes motivadoras da entrada e permanência dos médicos em hospitais com as características do INCA. Logo, haverá uma demanda permanente por treinamentos na medida em que a tecnologia evolua. Se feito de forma pouco transparente, o processo de escolha dos médicos que terão acesso aos treinamentos pode se transformar em fonte de desmotivação e discórdia.

Com o refinamento de técnicas e de ferramentas e com a evolução da curva de experiência, é provável que este novo método torne-se uma parte regular do arsenal cirúrgico do tratamento de câncer, inclusive nos serviços públicos de países emergentes. Nas condições atuais, para que isso ocorra, será necessária a definição clara e abrangente da tabela de remuneração do SUS. A criação da CONITEC e da DGITS e a institucionalização do seu papel nestas decisões poderão contribuir para que critérios mais efetivos de precificação sejam adotados no caso da cirurgia robótica.

Segundo os relatos obtidos, ainda serão necessários de dois a três anos para que os resultados da cirurgia apoiada por robô fiquem claros para o INCA. Durante o processo de análise dos resultados pelo MS é possível que os debates envolvam questões como:

i) a dependência crônica de um pequeno número de fabricantes internacionais de sistemas cirúrgicos robóticos e seus periféricos, cujas práticas tendem à concentração de mercado;

ii) a contribuição negativa das importações de equipamentos e insumos para o déficit do complexo industrial de saúde brasileiro, já muito elevado e, por consequência, alvo de ações públicas de mitigação.

Tais questões poderão induzir políticas públicas de desenvolvimento nacional destes equipamentos. O primeiro passo nesta direção poderá ser a atração, com acordos de transferência de tecnologia e de conteúdo nacional, de fabricantes, para o território nacional. Caberá à pesquisa futura avaliar os impactos dessas políticas para a inclusão no curto prazo da cirurgia robótica como um processo sistemático do SUS.

A próxima geração de sistemas cirúrgicos que está sendo desenvolvida deverá aumentar a capacidade de visualização e análise de imagens. A evidência da eficácia e segurança da cirurgia robótica e seus respectivos custos devem ser avaliados em uma ampla gama de procedimentos. Sendo assim, novos sistemas devem reduzir os custos de treinamento e da curva de aprendizagem dos médicos e novos projetos devem priorizar a reutilização de suprimentos. Uma importante questão para a pesquisa é analisar o futuro da tecnologia robótica na medicina, se em direção a uma complementariedade cada vez maior entre as habilidades do médico e a precisão da máquina ou se, a exemplo do que parece estar acontecendo em outros ramos, à completa substituição dos médicos por robôs cirúrgicos inteligentes.

\section{REFERÊNCIAS:}

Argyris, C., Putnam, R. \& Smith, D.M. (1985). Action science: action science concepts, methods and skills for research and intervention. Nova York: Jossey Bass.

Baek, S. et alli. (2012). Robotic versus conventional laparoscopic surgery for rectal cancer: a cost analysis from a single institute in Korea. World Journal of Surgery, 36(11), 2722-2729.

Ballantyne, G.H, \& Moll, F. (2003). The Da Vinci telerobotic surgical system: the virtual operative field and telepresence surgery. Surgical Clinics of North America, 83(6), 1293-1304.

Bardin, L. (1999). Análise de Conteúdo. Lisboa: Edições 70, LDA,.

Bell, M. \& Figueiredo, P.N. (2012). Innovation capability building and learning mechanisms in latecomer firms: recent empirical contributions and implications for research. Canadian Journal of Development Studies, 3(1), 1440. 
Button, V.L.S.N. \& Oliveira, E.J.V. (2012). Uma estratégia de desenvolvimento para o sistema nacional de inovação de produtos médicos. Revista Brasileira de Engenharia Biomédica, 28 (2), 124-139.

Calil, S.J. (2011). Gestão de tecnologias hospitalares. In: Vencina Neto, G.; Malik, A.M. Gestão em Saúde. 1a. ed. Rio de Janeiro: Guanabara Koogan.

Camarillo, D. B., Krummel, T. \& Salisbury, K.J. (2004). Robotic technology in surgery: past, present, and future. The American Journal of Surgery, 188 (4), 2-15.

Cecílio, L.C.O. (2009). A morte de Ivan Ilitch, de Leon Tolstói: elementos para se pensar as múltiplas dimensões da gestão do cuidado. Interface Comunicação Saúde Educação, 13(suppl.1), 545-55

Christensen, J. F. (2006). Whiter core competency for the large corporation in an open innovation world? In: Chesbrough, H.W, \& Vanhaverbeke, W. J. Open innovation: researching a new paradigm. New York: Oxford University Press.

Daniels, N. (2006). Toward ethical review of health system transformations. American Journal of Public Health, v (3), 447-451.

Dixon-Woods, M. et al. (2011). Problems and promises of innovation: why healthcare needs to rethink its love/hate relationship with the new. $B M J$ Quality \& Safety, 20(1), 147-151.

Ekedahl, M. \& Wengstrom, Y. (2008). Coping processes in a multidisciplinary healthcare team a comparison of nurses in cancer care and hospital chaplains. European Journal of Cancer Care, 7(1), 42-48.

Fichman, R G. (2000). The diffusion and assimilation of information technology innovations. In ZMUD R.B. (Dir.). Framing the domains of IT management: projecting the future through the past (pp. 105-127). Cincinnati Pinnaflex Educational Resources Inc.

Figueiredo, P. N. (2009). Gestão da inovação: conceitos, métricas e experiências de empresas no Brasil. Rio de Janeiro: LTC.

Figueiró, A.C., Frias, P.G. \& Navarro L.M. (2010). Avaliação em saúde: Conceitos básicos para as práticas nas instituições. In: (Eds) Samico, I., Felisberto, E., Figueiró, A.C., Frias, P.G. Avaliação em saúde: Bases conceituais e operacionais. Rio de Janeiro: Editora Medbook, 1-13

Freeman, C. \& Soete, L. (1997). The economics of industrial innovation. 3. ed. London: Frances Pinter.

Freire, R. P., Pitassi, C., Gonçalves, A.A., \& Schout, D. (2012). Gestão de equipamentos médicos: o papel das práticas de qualidade em um hospital de excelência brasileiro. Revista de Administração Hospitalar e Inovação em Saúde, 8(8), 28-41.

Frisse, M. C. (1999). The Business Value of Health Care Information Technology. Journal of the American Medical Informatics Association, SepOct, 6(5), 361-367.

Barbash, G.I., \& Glied, SA. . (2010). New technology and health care coststhe case of robot-assisted surgery. New England Journal of Medicine, 363, 701-704.

Gaia, G. et alli. A. (2010). Robotic-assisted hysterectomy for endometrial cancer compared with traditional laparoscopic and laparotomy approaches: a systematic review. Obstetrics \& Gynecology, 116(6), 1422-1431.

Nakib, G. et alli. G. (2013). Robotic assisted surgery in pediatric gynecology: promising innovation in mini invasive surgical procedures. Journal of Pediatric and Adolescent Gynecology, 6(1), e5-e7.

Gibilisco S. (1994). The McGraw-Hill illustrated encyclopedia of robotics \& artificial intelligence. New York: McGraw-Hill,

Giulianotti, P. C., et alli. (2003). Robotics in general surgery: personal experience in a large community hospital. Archives Surgery, 8(7), 777-784.

Gonçalves, A.A.; Pitassi, C. \& Moreno, V.Jr . (2014). The case of Inca's National Tumor Bank management system in Brazil. Revista de Gestão da Tecnologia e Sistemas de Informação (Online), 11(3), 549-568.

Gomes, P. (2011). Surgical robotics: reviewing the past, analyzing the present, imagining the future. Robotics and Computer-Integrated Manufacturing, 27(2), 261-266.

Hartley, J. (2005). Innovation in governance and public services: past and present. Public Money and Management, 25(1), .27-34.

Herzlinger, R. E. (2006). Why innovation in health care is so hard. Harvard Business Review, 84(5), 58-66.
Hughes, B. \& Wareham, J. (2010). Knowledge arbitrage in global pharma: a synthetic view of absorptive capacity and open innovation. Research \& Development Management, 40(3), 324-343.

Isaacson, W. (2014). The innovators: how a group of hackers, geniuses, and geeks created the Digital Revolution. New York: Simon \& Schuster

Lara, N. (2015). Diagnosis Related Groups (DRG's) e seus efeitos sobre os custos e a qualidade dos serviços hospitalares. Instituto de Estudos de Saúde Complementar, Textos para Discussão, 54

Levaggi, R., Moretto, M. \& Rebba, V. (2009). Investment decisions in hospital technology when physicians are devoted workers. Economics of Innovation and New Technology, 18(5), 487-512.

Maggio, L.A et alli. (2016). Challenges to learning Evidence-Based Medicine and educational approaches to meet these challenges: A qualitative study of selected EBM curricula in U.S. and Canadian medical schools. Academic Medicine, 91(1),101-106.

Miles, M.B., Huberman. M.A. \& Saldaña, J. (2014). Qualitative data analysis. A methods sourcebook. London: SAGE Publications.

Ministério da Saúde (2009). Diretrizes metodológicas: estudos de avaliação econômica de tecnologias em saúde.

Ministério de Saúde (2010). Política Nacional de Gestão de Tecnologias em Saúde. Secretaria de Ciência, Tecnologia e Insumos Estratégicos.

Moraz, G. et alli. (2015). Estudos de custo-efetividade em saúde no Brasil: uma revisão sistemática. Ciência \& Saúde Coletiva, 20(10), 3211-3229.

Nof, S.Y. \& Hall, N. G. (2007). Handbook of industrial robotics. Second Edition. New Jersey: John Wiley \& Sons, Inc.

Kaplan, R.S. \& Porter, M.E. (September 2011). How to solve the cost crises in health care. Harvard Business Review, 89(9), 47-64.

O'Donnell, .R.R. (2014). New models for chronic disease management in the United States and China. Family Medicine and Community Health, 2(4):1319.

Organização para a Cooperação e Desenvolvimento Econômico-OCDE (2007). Manual de Oslo - Diretrizes para a coleta e interpretação de dados sobre Inovação. $3^{\text {a }}$ ed., Tradução FINEP, Recuperado de http://download.finep.gov.br/imprensa/oslo2.pdf.

Orvieto M.A. et alli. (2011). Robotic technologies in surgical oncology training and practice. Surgical Oncology, 20(3), 203-209.

Porter, M.E. \& Lee, T.H. (October 2103). The strategy that will fix health care. Harvard Business Review, Disponível em https://hbr.org/2013/10/thestrategy-that-will-fix-health-care. Acesso em abril de 2015.

Powell, W.W.; White, D; R.; Koput, K.W. \& Owen-Smith, Jason. (2005) Network dynamics and field evolution: the growth of interorganizational collaboration in the life sciences. American Journal of Sociology, 110(4), 1132-1205.

Queiroz, D.T., Vall, J., Souza, J.M.A, Vieira, N.F.C. (2007). Observação participante na pesquisa qualitativa: Conceitos e aplicações na área de saúde. Revista de Enfermagem da UERJ, 15(2), 276-283.

Rogers, E. M. (2003). Diffusion of innovations. New York: Free Press.

Schumpeter, J. (1934). The theory of economic development. Cambridge: Harvard UP.

Silva, L. K. (2003). Avaliação tecnológica e análise de custo-efetividade em saúde: a incorporação de tecnologias e a produção de diretrizes clínicas para o SUS. Ciência \& Saúde Coletiva, 8(2), 501-520.

Thakur, R., Hsu, S. H.Y \& Fontenot, G. (2012). Innovation in healthcare: Issues and future trends. Journal of Business Research, 65(4), 562-569.

Tidd, J., Bessant, J. \& Pavitt, K. (2008). Gestão da inovação. Porto Alegre: Bookman.

Varkey, P., Horne, A. \& Bennet, K.E. (2008). Innovation in health care: a primer. American Journal of Medical Quality, 23(5), 382-388.

Yin, R. K. (2005). Estudo de caso: planejamento e métodos. Porto Alegre: Bookman.

Windrum, P., Garcia-Goñi, M. \& Fairhurst, E. (2010). Innovation in public health care: diabetes education in the UK. In: Gallouj F. \& Djellal F. The Handbook of Innovation and Services: a multi-disciplinary perspective. Cheltenham, UK: Edward Elgar. 